\title{
On the evolution of massive close binaries
}

\author{
Norbert Langer ${ }^{1}$, Stephan Wellstein ${ }^{2}$, and Jelena Petrovic ${ }^{1}$ \\ ${ }^{1}$ Sterrekundig Instituut, Universiteit Utrecht, \\ Princetonplein 5, NL-3584CC Utrecht, Nederland \\ ${ }^{2}$ Lehrstuhl Astrophysik, Institut für Physik, Universität Potsdam, \\ Am Neuen Palais 10, D-14414 Potsdam, BRD
}

\begin{abstract}
We discuss which fraction of the matter flowing to the companion during a Roche lobe overflow phase can actually be accreted by the secondary star. Employing new evolutionary models for massive close binaries which include the effects of rotation for both components as well as angular momentum accretion and spin-orbit coupling, we propose a physical model to calculate the accretion efficiency in Case A and B systems. We provide examples showing that both cases, high and low accretion efficiency, do occur within these models, as it seems required by observed post-mass transfer systems. Furthermore, we discuss late evolutionary stages of such binaries, with emphasis on the formation of compact objects: what are their spin rates, which systems can produce black holes, which gamma-ray bursts?
\end{abstract}

\section{Introduction}

The evolution of a single star depends on its fundamental initial parameters: mass, chemical composition and - as re-emphasised recently - angular momentum (Heger \& Langer 2000; Meynet \& Maeder 2000). For a star in a close binary there are at least two more initial parameters to be considered: the stellar mass ratio and the orbital period. Is binary evolution an important enough issue to justify the effort to cope with this huge parameter space?

The answer is clearly: Yes! Some of the most exciting cosmic phenomena occur exclusively in binaries: Type Ia supernovae, the main producers of iron and cosmic yardstick to measure the accelerated expansion of the universe. Gammaray bursts - which current stellar models with rotation preclude to occur in single stars (Heger, Langer \& Woosley 2000; Fryer, these Proceedings) - are the most powerful explosions in the universe and trace star formation to its edge. And black holes, even though also formed by single stars, need a companion to become 'visible'.

A second reason to study binary evolution is their use as laboratory for stellar physics. Effects of rotation - important for single stars but not yet completely understood - occur much stronger in the accreting components of close binaries. The same holds for the physics of the so called $\Omega$-limit (Langer 1997). In order to investigate these effects, we need binary evolution models which include a detailed treatment of spin and orbital angular momentum. Some first such models are discussed below, shedding new light on the question: which 
Table 1. Clues on accretions efficiencies from observed binaries.

\begin{tabular}{llccc}
\hline \hline object & $\begin{array}{l}\text { spectral } \\
\text { type }\end{array}$ & $\begin{array}{c}\text { orb. period } \\
(\mathrm{d})\end{array}$ & masses/ratio & accretion? \\
\hline${\text { V729 } \mathrm{Cyg}^{a}}^{a}$ & $\mathrm{O}+\mathrm{O}^{b}$ & 6.6 & $q=3.5$ & yes \\
${\text { Wray } 977^{c}}^{\mathrm{BI}}$ & $\mathrm{BIN}$ & 44 & $40 \mathrm{M}_{\odot}+1.4 \mathrm{M}_{\odot}$ & yes \\
3 systems $^{d}$ & WNE+O & $\sim 7$ & $q \simeq 0.5$ & no $^{e}$ \\
$4 \mathrm{U} 1700-37^{f}$ & O6I+NS/BH & 3.4 & $58 \mathrm{M}_{\odot}+2.4 \mathrm{M}_{\odot}$ & no $^{e}$ \\
\hline \hline
\end{tabular}

Notes: $a$ : Bohannan \& Conti, $1976 ; b$ : both components have the same visual magnitude; $c$ : Kaper et al. 1995, Wellstein \& Langer 1999; $d$ : Petrovic \& Langer 2003; $e$ : meaning: $10 \%$ or less; $f$ : Clark et al. 2002.

fraction of the mass transferred during a Roche lobe overflow phase can be accreted by the secondary star?

\section{Can massive stars accrete from a companion?}

Table 1 lists parameters of four different kinds of massive close binary systems which give opposite answers. The two O-type stars in the Case A ( $c f$. Wellstein et al. 2001, for a definition of the various mass transfer cases) binary V 729 Cyg have a mass ratio of 3.5, but the same spectral type and visual flux. Clearly, an initial mass ratio close to 1 is required to get close to the observed current mass ratio. However, as during Case A the primary star loses just about half of its mass, a mass ratio of at most 2 could be produced were the secondary not allowed to accrete. Another system showing strong evidence for accretion is the massive X-ray binary Wray 977; it would require that stars of $\gtrsim 40 \mathrm{M}_{\odot}$ form neutron stars to explain this system without accretion (Wellstein \& Langer 1999). Several Galactic short period WNE+O binaries, on the other hand, can not be understood had the O-type star accreted substantial amounts from the WNE progenitor ( $c f$. Petrovic \& Langer 2003). While those might have formed through Case C evolution - for which no accretion is expected - the key Xray binary $4 \mathrm{U} 1700-37$ has such a short period that a major accretion phase can be excluded. However, as Case $\mathrm{C}$ evolution would lead to a compact object much more massive than $2.4 \mathrm{M}_{\odot}$, Case B evolution is most likely here (Clark et al. 2002). We conclude that some massive Case A and B binaries encompass accretion, others do not. What makes the difference?

\section{Binary models with rotating components}

We have constructed binary evolution models using the code of Wellstein et al. (2001), but including the physics of rotation as in the single star models of Heger et al. (2000) for both components. In addition, spin-orbit coupling according to Zahn (1977) has been added, and rotationally enhanced winds are implemented as in Langer (1998). The specific angular momentum of the accreted matter is assumed to be that of Kepler rotation at the stellar equator in the case of disk accretion, and determined by integrating the equation of motion 


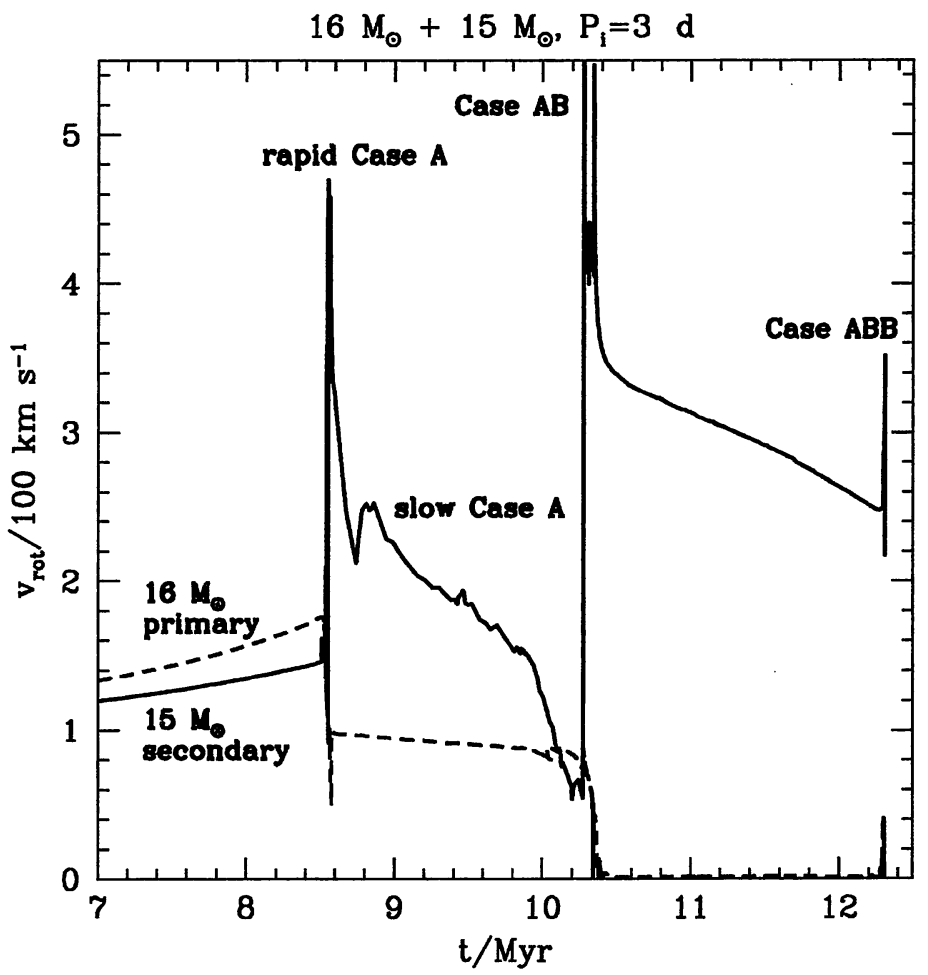

Figure 1. Equatorial rotation velocity for primary (dashed line) and secondary (solid line) component of a $16+15 \mathrm{M}_{\odot}$ system with an initial orbital period of $3 \mathrm{~d}$ as function of time, starting at an age of $7 \mathrm{Myr}$, i.e., well before the onset of mass transfer, until the end of Case ABB mass transfer, which corresponds to the time of the supernova explosion of the primary. The four different mass transfer phases which occur in this system are indicated; except for the slow Case A mass transfer they occur on the thermal time scale of the primary star ( $c f$. also Figure 2).

of a test particle in the Roche potential in case the accretion stream impacts directly on the secondary star.

Figure 1 shows the evolution of the equatorial rotation velocity in a system starting out with a $16 \mathrm{M}_{\odot}$ and a $15 \mathrm{M}_{\odot}$ star in a 3 day orbit. The initial rotational velocity of both stars is unimportant since they evolve into rotation which is synchronous with the orbital revolution. Each of the three thermal time scale mass transfer phases through which this system evolves (rapid Case A, Case AB, and Case ABB; cf. Wellstein et al. 2001) lead to a strong spin-up of the secondary star and an equally drastic spin-down of the primary (see Langer 1998, for the spin-down effect).

The accretion induced spin-up can bring the secondary close to critical rotation and thus to strong rotationally enhanced mass loss. The difference between the mass overflow rate $-\dot{M}_{1}$ and the wind mass loss rate of the secondary is the net accretion rate $\dot{M}_{2}$ of the secondary star. Figure 2 shows that during much of the rapid Case A mass transfer, it is $-\dot{M}_{1}=M_{2}$. This is possible due to two 


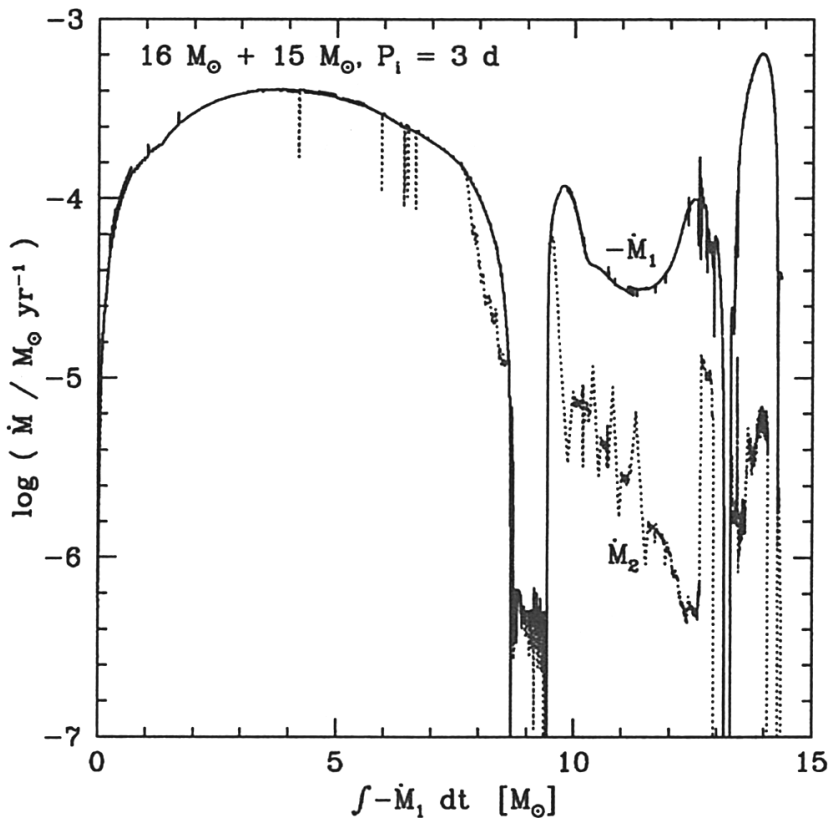

Figure 2. For the $16 \mathrm{M}_{\odot}+15 \mathrm{M}_{\odot}$ system with an initial orbital period of 3 days: mass transfer rate $\left(-\dot{M}_{1}\right.$; full drawn line) and mass accretion rate of the secondary $\left(\dot{M}_{2}\right.$; dotted line) as function of the total amount of mass which has already been transferred. Four discrete mass transfer phases can be distinguished: rapid Case A $\left(-\dot{M}_{1} \gtrsim 10^{-4} \mathrm{M}_{\odot} \mathrm{yr}^{-1}, \Delta M_{1} \simeq 8.7 \mathrm{M}_{\odot}\right)$; slow Case A $\left(-\dot{M}_{1} \gtrsim 10^{-7} \mathrm{M}_{\odot \mathrm{yr}^{-1}}, \quad \Delta M_{1} \simeq 0.8 \mathrm{M}_{\odot}\right) ; \quad$ Case $\mathrm{AB} \quad\left(-\dot{M}_{1} \gtrsim 10^{-5} \mathrm{M}_{\odot} \mathrm{yr}^{-1}\right.$, $\left.\Delta M_{1} \simeq 3.8 \mathrm{M}_{\odot}\right) ;$ and Case ABB $\left(-\dot{M}_{1} \gtrsim 10^{-4} \mathrm{M}_{\odot} \mathrm{yr}^{-1}, \Delta M_{1} \simeq 1.2 \mathrm{M}_{\odot}\right)$.

factors: $(i)$ during direct impact accretion, the specific angular momentum of the accreted material is only a fraction of the respective Kepler angular momentum; (ii) as the secondary star fills a significant fraction of its Roche volume, tidal coupling removes part of the accreted angular momentum and feeds it into the orbit.

As the orbit widens significantly during the later evolution, the specific angular momentum of the accreted matter increases and tidal forces weaken. Thus, while the accretion efficiency is close to 1 during Case A, it is less than 0.1 later, resulting in a time average over the whole evolution of 0.67 . For systems which start out with a wider orbit, we find a low accretion efficiency throughout (Figure 3). For $16 \mathrm{M}_{\odot}+15 \mathrm{M}_{\odot}$ systems, we find a critical initial period of $\sim 8 \mathrm{~d}$ beyond which the secondary accretes only little.

These models imply that, despite the angular momentum problem for the accretion star (Packet 1981), quasi-conservative evolution of massive close binaries is possible. However, already in early Case B systems, the accretion efficiency may be strongly reduced compared to binary models without rotation.

Further obvious conclusions from the results presented above are: The primaries of massive close binaries may produce slowly spinning neutron stars or black holes $(P \simeq 1 \mathrm{~s})$. And as the collapsar model for $\gamma$-ray bursts requires high 


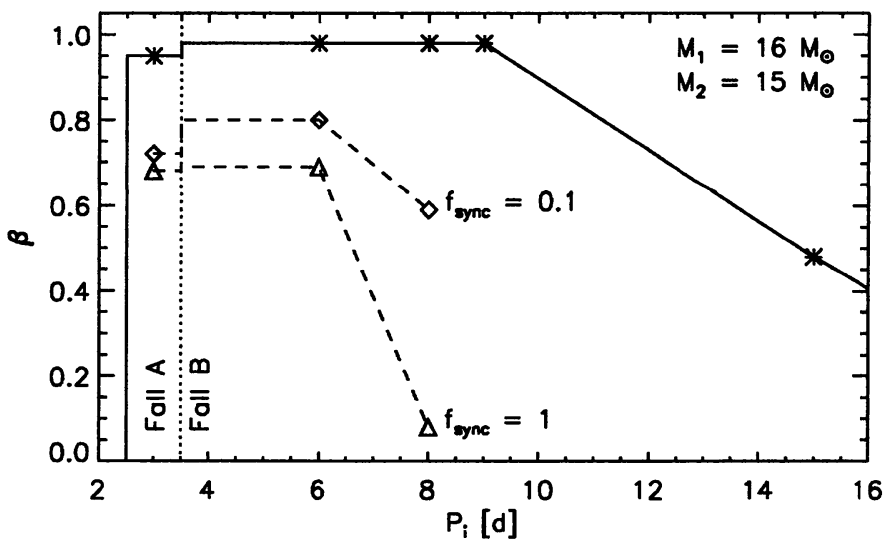

Figure 3. Time averaged mass accretion efficiency $\beta$ for various binary evolution sequences (symbols) and different physical assumptions, as function of the initial orbital period for $16 \mathrm{M}_{\odot}+15 \mathrm{M}_{\odot}$ systems. Triangles mark systems which have been calculated using rotational physics and the tidal model as proposed by Zahn (1977), which in systems marked by squares has been assumed to be one order of magnitude more efficient. Star symbols designate models by Wellstein et al. (2001) which have been computed without rotational physics and assumed to evolve conservatively unless a contact situation occurs. The dotted vertical line separates Case A systems (to the left) from Case B systems.

angular momenta, spun-up secondaries may provide a viable $\gamma$-ray burst progenitor model.

\section{How to form black hole binaries}

While single stars more massive than $\sim 25 \mathrm{M}_{\odot}$ are supposed to form black holes (cf. Fryer, these Proceedings), it is a challenge to binary evolution modellers to find a way to make black hole binaries - binary systems consisting of a nondegenerate star and a black hole. The reason is that the initially more massive star, which is supposed to end up as black hole, undergoes tremendous mass loss (cf. Figure 2, and see Wellstein \& Langer 1999).

Brown, Lee \& Bethe (1999) suggested the Case C channel to form the Galactic low mass BH binaries - a low mass star in close orbit with a black hole of typically $6 \mathrm{M}_{\odot}$ (cf. Orosz, these Proceedings), i.e., starting with a wide system consisting of a (say) $25 \mathrm{M}_{\odot}$ and a $5 \mathrm{M}_{\odot}$ star. This suggestion is strongly supported by the massive post-Case B.X-ray binary 4U 1700-37 (cf. Table 1): it shows that in Case B systems, even a $60 \mathrm{M}_{\odot}$ primary forms a compact object of only $2.4 \mathrm{M}_{\odot}$ (Clark et al. 2002).

The problem of how wide the Case $C$ channel is for such massive stars is difficult. Current single star models suggest no significant radius increase late during or after core helium burning at solar metallicity. On the other hand, Smartt's abundance analysis of Galactic supergiants (these Proceedings) suggests that they do not undergo 'blue loop' evolution - which contradicts the current models. The problem is less severe at low metallicity, where many 
models predict a gradual radius increase during core hydrogen burning. It is even harder to produce Cyg X-1 type $\mathrm{BH}$ binaries, with black hole masses as high as $14 \mathrm{M}_{\odot}$. Brown et al. (2001) argue that this requires Case $\mathrm{C}$ evolution and an upturn of the initial-final mass relation for single stars above $\sim 60 \mathrm{M}_{\odot}$, as predicted by Langer (1987).

\section{References}

Bohannan, B., Conti, P.S. 1976, ApJ 204, 797

Brown, G.E., Lee, C.-H., Bethe, H.A. 1999, New Astron. 4, 313

Brown, G.E., Heger, A., Langer, N., et al. 2001, New Astron. 6, 457

Clark, J.S., Goodwin, S.P., Crowther, P.A., Kaper, L., Fairbairn, M., Langer, N., Brocksopp, C. 2002, A\&A 392, 909

Heger, A., Langer, N. 2000, ApJ 544, 1016

Heger, A., Langer, N., Woosley, S.E. 2000, ApJ 528, 368

Kaper, L., Ruymaekers, E., van den Heuvel, E.P.J., et al. 1995, A\&A 300, 446

Langer, N. 1987, A\&A (Letters) 171, L1

Langer, N. 1997, in: A. Nota \& H. Lamers (eds.), Luminous Blue Variables: Massive Stars in Transition, ASP-CS 120, 332

Langer, N. 1998, A\&A 329, 551

Meynet, G., Maeder, A. 2000, A\&A 361, 101

Packet, W. 1981, A\&A 102, 17

Petrovic, J., Langer, N. 2003, these Proceedings

Wellstein, S., Langer, N. 1999, A\&A 350, 148

Wellstein, S., Langer, N., Braun, H. 2001, A\&A 369, 939

Zahn, J.-P. 1977, A\&A 57, 383

\section{Discussion}

CherepashCHUK: Including eccentricity in the initial orbit in your calculation could help with formation of massive black holes in close binaries without case C evolution, because after tidal dissipation a circularisation of the orbit separation between the components is decreasing. It can increase the mass exchange rate.

LANGER: In our Case A and early Case B models, the tides lead to circularisation and synchronisation before the first mass transfer. For later systems we expect this to happen very early during the first mass transfer phase. So, I currently don't see how an initial eccentricity could help.

FOELLMI: The problem of observations of WR+OB binaries is that we obtain a snapshot of the evolution and it is hard to know effects of a previous interacting binary history. There are two interesting systems in the SMC with a similar $15 \mathrm{~d}$ period: SMC-WR8 (WC4+O4, for which the spectral types point to a rejuvination process) and SMC-WR3 (WN3h+O9, no evidence for rejuvenation and hence accretion). We did find hydrogen in the WR wind. Can we use the $\mathrm{H}$ abundance in the WR to test a possible previous occurrence of a RLOF?

LANGER: Thanks for adding further examples of tests of the accretion efficiency in massive close binaries; those are urgently needed to constrain our theories. The presence of hydrogen in a WR wind cannot rule out that RLOF has occurred, as RLOF does not remove hydrogen completely in the mass losing component of the binary. 